\title{
Aesthetic Correction of Gingival Pigmentation Using Different Techniques
}

\author{
Subhapriya Mandal ${ }^{1}$, Sharanya Bose ${ }^{2}$, Poulomi Choudhuri ${ }^{3}$, Himadri Chakrabarty ${ }^{4}$, Abhijit Chakrabarty ${ }^{5}$ \\ ${ }^{1}$ Department of Periodontics, Guru Nanak Institute of Dental Sciences and Research, Kolkata, West Bengal, India. \\ ${ }^{2}$ Department of Periodontics, Guru Nanak Institute of Dental Sciences and Research, Kolkata, West Bengal, India. \\ ${ }^{3}$ Department of Periodontics, Guru Nanak Institute of Dental Sciences and Research, Kolkata, West Bengal, India. \\ ${ }^{4}$ Department of Periodontics, Guru Nanak Institute of Dental Sciences and Research, Kolkata, West Bengal, India. \\ ${ }^{5}$ Department of Periodontics, Guru Nanak Institute of Dental Sciences and Research, Kolkata, West Bengal, India.
}

\section{INTRODUCTION}

Men and women of the present generation are extremely conscious of how they present themselves before the world and have been giving enormous importance to their aesthetics in every facet to augment their personality. An appealing smile boosts one's individuality. The harmony of the smile is attributable to the contour, colour, and position of the teeth in conjunction with the gingival tissue. Hence undesirable gingival pigmentation can be a major drawback in the aesthetics of the patient Gingival pigmentation is defined as the discoloration of the gingiva due to lesions analogous with extrinsic as well as intrinsic factors.[1] The five primary pigments melanin, melanoid, oxyhaemoglobin, reduced haemoglobin and carotene are the main causative factors of depigmentation.[2]

The brown melanin pigment is the most prevalent pigment which contributes to the endogenous pigmentation of the gingiva. Melanin pigmentation results from melanin granules which are produced by melanoblasts.[3] Melanin which is a nonhaemoglobin-derived pigment, is formed by cells called melanocytes and these melanocytes are dendritic cells of neuroectodermal origin in the basal and spinous layers. ${ }^{[4]}$ It is seen that the attached gingivae is $27.5 \%$ times more pigmented than any other intraoral tissues. Melanin pigmentation is mostly localized at the anterior labial gingiva.[5] It is often noted that even environmental factors such as tobacco smoking contribute to the gingival hyperpigmentation in both active and passive form. ${ }^{[6-9]}$ The colour of gingiva is influenced by the ethnicity and age and has no sexual predilection.[10]

A beautiful smile enhances one's confidence. This case series enlightens us regarding the various depigmentation methods for enhancing the smile. Gingival pigmentation is caused mainly due to abnormal accumulation of melanin in the gingival tissues. The study describes the various methods like scalpel technique (using BP blade), LASER Doctor Smile Diode LASER and electrocautery (Servotome). Healing was satisfactory in all three cases. Gingival depigmentation using LASER technique was found to be superior when compared to Scalpel and Electrocautery techniques.
Corresponding Author:

Sharanya Bose,

\#92, Uday Shankar Sarani,

Golf Club Road, Tollygunge-700033,

Kolkata, West Bengal, India.

E-mail: sharonbasu@gmail.com

DOI: $10.14260 / \mathrm{jemds} / 2020 / 237$

Financial or Other Competing Interests: None.

How to Cite This Article:

Mandal S, Bose S, Choudhuri P, et al. Esthetic correction of gingival pigmentation using different techniquescase reports. J. Evolution Med. Dent. Sci. 2020;9(13):1102-1105, 10.14260/jemds/2020/237

Submission 10-02-2019, Peer Review 10-03-2020, Acceptance 12-03-2020, Published 30-03-2020.

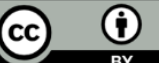




\section{Case 1}

A young female patient, aged 22 years visited the Department of Periodontics, GNIDSR, Kolkata complaining of heavily pigmented gums. On examination, the DOPI score was evaluated to be 3 . The patient, who demonstrated a high smile line, exposed a deeply pigmented gingiva from first premolar to first premolar [Figure 1]. Keeping in mind the patient's concern, it was decided to go ahead with LASER depigmentation [Figure 2] procedure. The entire course of procedure that was to be conducted was explained to the patient and a written consent was obtained before commencing with the procedure. Routine oral hygiene procedures were carried out. In the maxillary anterior region infiltration was administered. After achieving local anaesthesia, the diode LASER was used in the affected region (in contact mode). The LASER ablation was carried out using a hand piece with fiber optic filament, 300 micrometer in diameter at $1.0 \mathrm{~W}$. [Figure $3 \& 4$ ] The complete procedure was carried out in contact mode and the tip was moved in light brushing strokes and was kept in motion all the time. The vestige ablated tissues were eliminated using a sterile cotton roll already moistened with saline solution. There was no complain of pain on patient's behalf during and after the procedure, neither there was any bleeding complications during and post the procedure. [Figure $5 \& 6$ ] The patient was prescribed to apply Capsule Evion $400 \mathrm{mg}$ topically on the operated site thrice daily for five days. One day after the completion of the procedure, there was no complaint of any pain or bleeding on patient's behalf. The ablated wound healed well after one week. Post three months the gingiva appeared moderately pink and healthy and it was well epithelialized. [Figure 7]

\section{Case 2}

A 25 years old young male patient, complaining of black gums, visited the Department of Periodontics, GNIDSR, Kolkata. [Figure 8] The patient had a DOPI score of 3 revealing a high smile line. Considering the demands of the patient, the process of depigmentation was decided to carry out using electrocautery. [Figure 9] A loop electrode was used for deepithelializing the gingiva and it was used in light brushing stroke, keeping the tip in motion all the time. The operator kept in mind that the tip was in contact with the tissue for a very brief period of time as keeping the tip at one place could lead to immense heat escalation (lateral heat accumulation) and eventually destruction of the tissue. [Figure 10 \& 11] The tip was cleaned after every use, using a saline moistened cotton roll to free the tip of all the debris. The patient was asked to follow the basic oral hygiene practice. After 1 week the patient was recalled and on examination the newly formed epithelium did not display any pigmentation. [Figure 12 \& 13] After 6 weeks the gingiva appeared pale pink in colour and was apparently healthy. [Figure $14 \& 15]$

\section{Case 3}

A 29-year-old young male patient visited the visited the Department of Periodontics, GNIDSR, Kolkata with a request of treating his esthetically incompetent black gums. Examination revealed heavily pigmented gingiva from canine to canine with DOPI score of 3 and a high smile line was also documented. [Figure 16] It was decided to go ahead with scalpel surgical depigmentation procedure. Local anaesthetic infiltration (2\% lignocaine) was administered in the maxillary anterior region from the right first premolar to left first premolar. Once adequate local anesthesia was achieved, the pigmented gingival epithelium and underlying layer of connective tissue is surgically removed using Bard Parker blade number 15 and 11. [Figure 17] It was taken due care that no tissues were torn while doing the surgery and neither any pigmented remnants were left over the denuded area. [Figure 18] After the goal of adequate hemostasis was achieved, periodontal pack was placed over the surgical area. [Figure 19] The healing process was observed to be normal after 7 days and the patient did not face any discomfort. [Figure $20 \& 21$ ] After one month, re-epithelialization was complete and the healing was adequate and acceptable. [Figure 22]

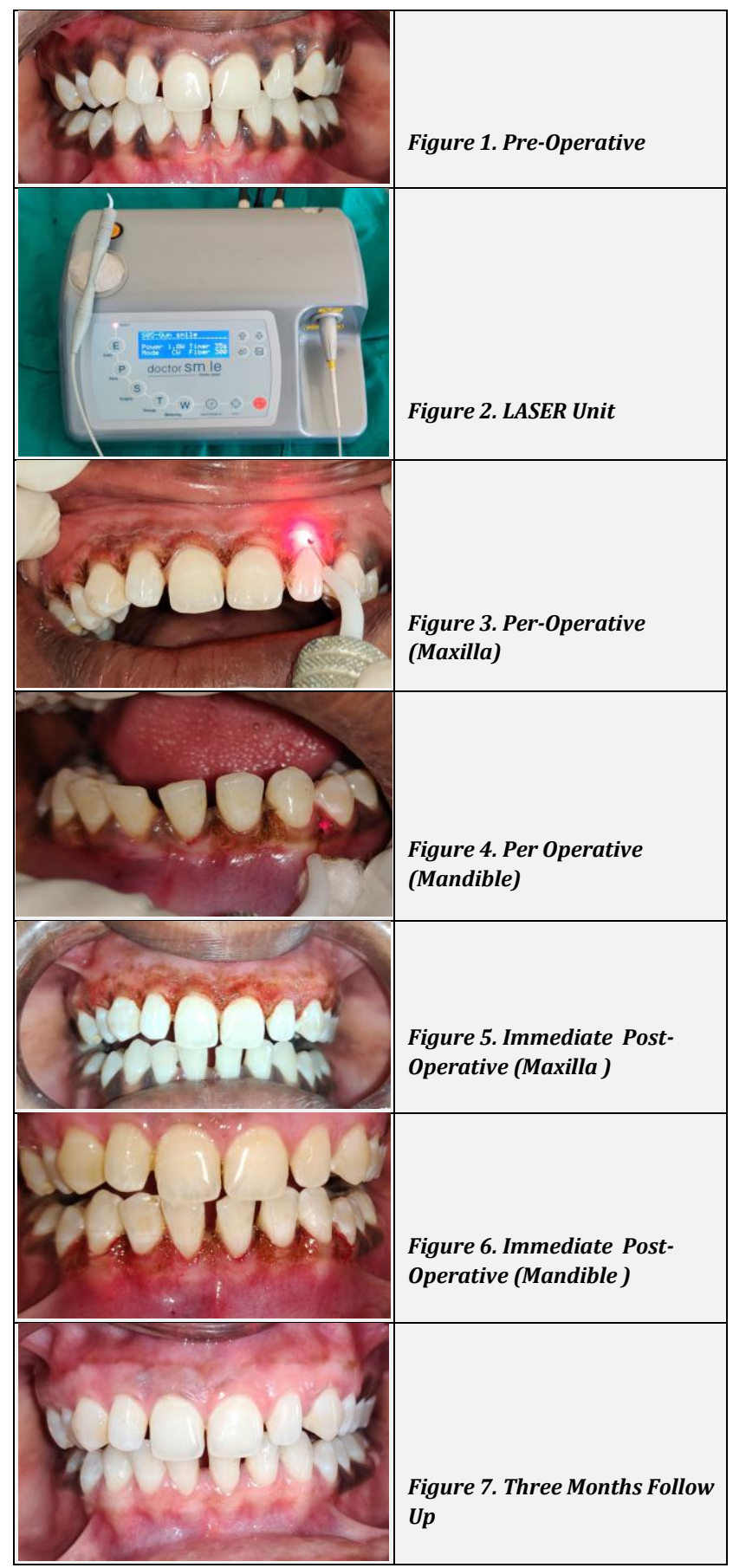




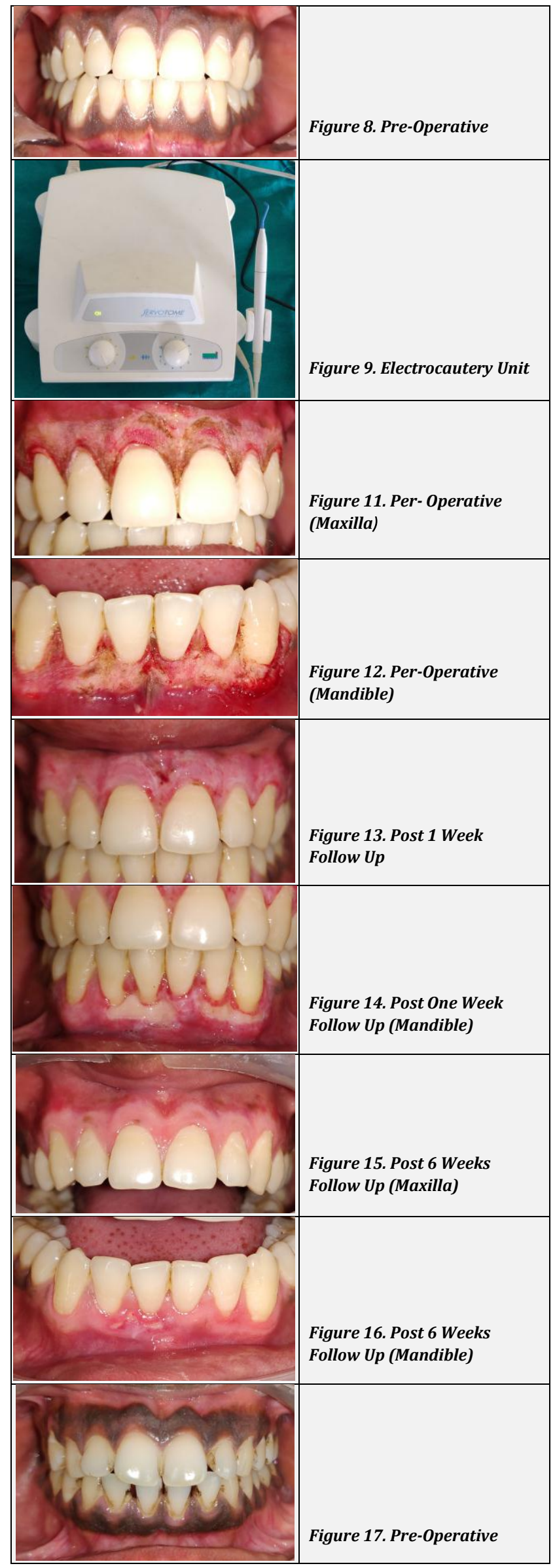

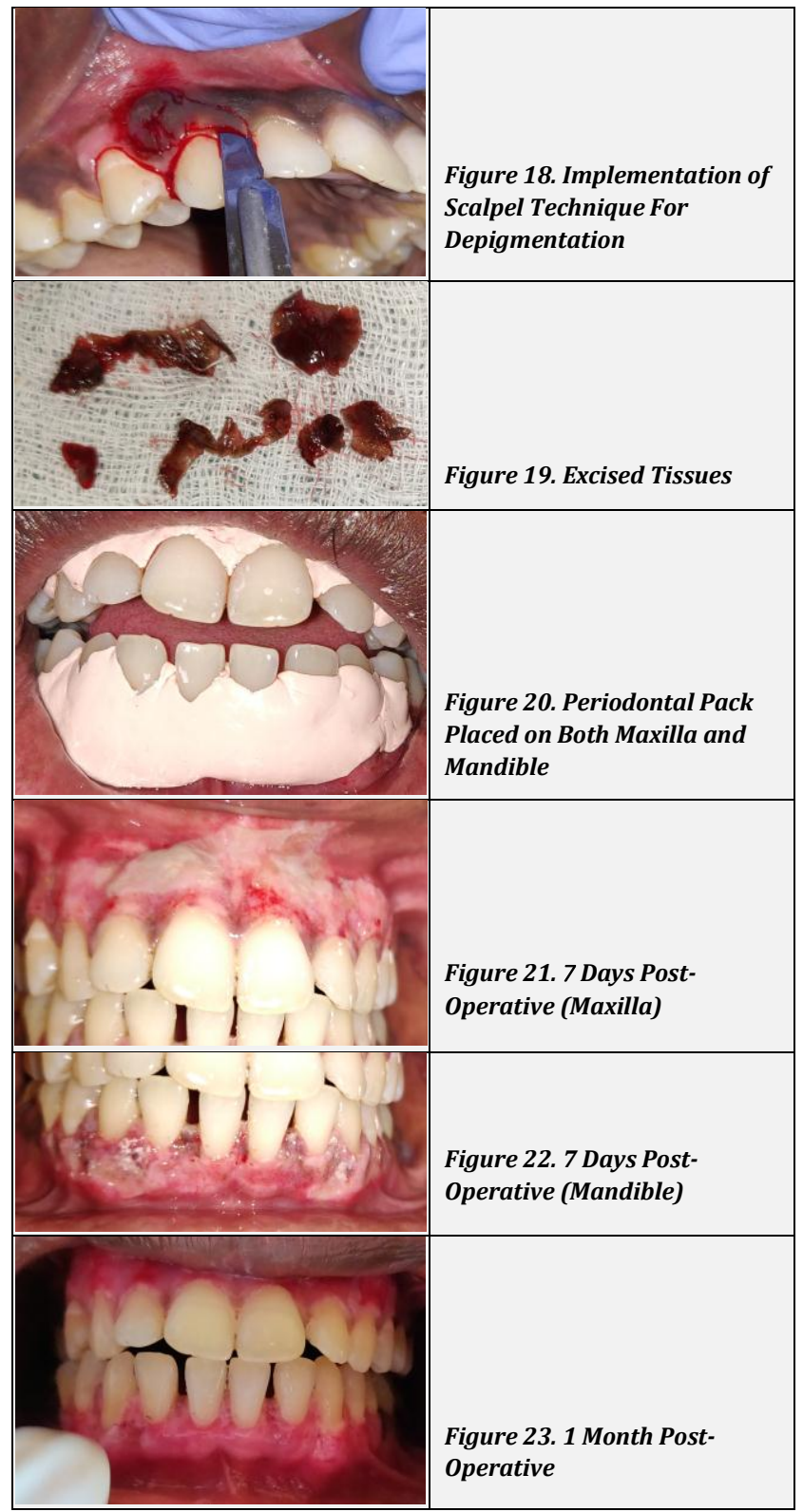

\section{CONCLUSIONS}

As gingival hyperpigmentation is considered to be unaesthetic by patients, innumerable methods can be adopted to treat the same. The loopholes of older techniques like scalpel surgery, electrosurgery and gingival abrasion using bur are that they are invasive, tedious, very painful, often requiring a periodontal dressing and common postoperative complications. The newer techniques, namely LASER and cryosurgery, on the other hand are more acceptable and usually the postoperative complications are minimal. All the depigmentation procedures yielded good result. But the LASER technique stands out as it is extremely patient friendly, noninvasive and has minimal post-operative complications. 


\section{REFERENCES}

[1] Kauzman A, Pavone M, Blanas N, et al. Pigmented lesions of the oral cavity: review, differential diagnosis, and case presentations. J Can Dent Assoc 2004;70(10):682-3.

[2] Steigmann S. Treatment of melanin-pigmented gingiva and oral mucosa by CO2 laser. Oral Surg Oral Med Oral Pathol Oral Radiol Endod 2000;90(1):14-5.

[3] Patel KA, Patil SS, Agrawal C, et al. Gingival depigmentation: Case series. Int J Appl Dent Sci 2015;1(2):37-9.

[4] Eid HA, Syed S, Soliman ANM. The role of gingival melanin pigmentation inflammation of gingiva, based on genetic analysis. J Int Oral Health 2013;5(4):1-7.

[5] Ciçek Y, Ertaş U. The normal and pathological pigmentation of oral mucous membrane: a review. J Contemp Dent Pract 2003;4(3):76-86.

[6] Multani S. Interrelationship of smoking, lip and gingival melanin pigmentation and periodontal status. Addict Health 2013;5(1-2):57-65.

[7] Hanioka T, Tanaka K, Ojima M, et al. Association of melanin pigmentation in the gingiva of children with parents who smoke. Pediatrics 2005;116(2):e186-90.

[8] Tadakamadla J, Kumar S, Nagori A, et al. Effect of smoking on oral pigmentation and its relationship with periodontal status. Dent Res J (Isfahan) 2012;9(Suppl 1):S112-4.
[9] Moravej-Salehi E, Moravej-Salehi E, Hajifattahi F. Relationship of gingival pigmentation with passive smoking in women. Tanaffos 2015;14(2):107-14.

[10] Ho DK, Ghinea R, Herrera LJ, et al. Color range and color distribution of healthy human gingiva: a prospective clinical study. Sci Rep 2015;5:18498.

[11] Malhotra S, Sharma N, Basavaraj P. Gingival esthetics by depigmentation. J Periodontal Med Clin Pract 2014;1(1):79-84.

[12] Grover HS, Dadlani H, Bhardwaj A, et al. Evaluation of patient response and recurrence of pigmentation following gingival depigmentation using laser and scalpel technique: a clinical study. J Indian Soc Periodontol 2014;18(5):586-92.

[13] Elavarasu S, Thangavelu A, Alex S. Comparative evaluation of depigmentation techniques in split-mouth design with electrocautery and laser. J Pharm Bioallied Sci 2015;7(Suppl 2):S786-90.

[14] Deepak P, Sunil S, Mishra R, et al. Treatment of gingival pigmentation: A case series. Indian J Dent Res 2005;16(4):171-6.

[15] Dummet CO, Barens G. Oromucosal pigmentation: an updated literary review. J Periodontol 1971;42(11):72636.

[16] Liebart MF, Caroline FD, Alain S, et al. Smile line and periodontium visibility. Perio 2004;1(1):17-25. 